\title{
ReaR
}

ISNN 19894090

Revista electrónica de AnestesiaR

Febrero 2022

ARTÍCULOS DE REVISIÓN

\section{Bases de la interacción corazón-pulmón en Anestesiología y Cuidados Intensivos.}

Paz Martin D (1), Serna Gandía M (2), Tovar Doncel MS (1), García-Montoto Pérez F (3).

(1) Complejo Hospitalario de Toledo.

(2) Hospital Marina Salud Denia, Alicante.

(3) Complejo Hospitalario Universitario de Cáceres.

\section{Resumen}

La interacción entre el corazón y los pulmones es uno de los ritmos básicos de la vida y fluctúa con la frecuencia del latido cardiaco y de la respiración. El conocimiento de este conjunto de interacciones mecánicas, neuronales y humorales resulta fundamental en la práctica de la Anestesiología y los Cuidados Intensivos. Por una parte, son la base de la monitorización hemodinámica funcional y ayudan a entender sus limitaciones, por otra, permiten profundizar en la comprensión de la fisiología del paciente críticamente enfermo y, muy especialmente, en los efectos de la ventilación mecánica en sujetos con reserva cardiovascular comprometida.

\section{Introducción}

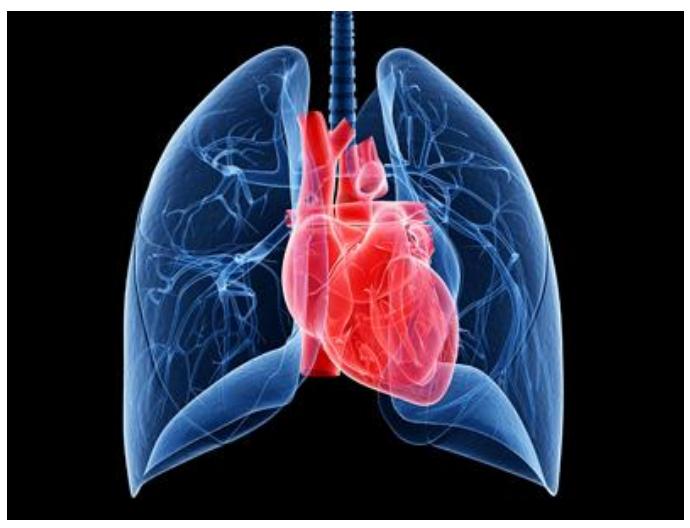

La interacción entre el corazón y los pulmones es uno de los ritmos básicos de la vida y fluctúa con la frecuencia del latido cardiaco y de la respiración. El conocimiento de este conjunto de interacciones mecánicas, neuronales y humorales resulta fundamental en la práctica de la Anestesiología y los Cuidados Intensivos. Por una parte, son la base de la monitorización hemodinámica funcional $\mathrm{y}$ ayudan a entender sus limitaciones, por otra, permiten profundizar en la comprensión de la fisiología del paciente críticamente enfermo y, muy especialmente, en los efectos de la ventilación mecánica en sujetos con reserva cardiovascular comprometida.

La interacción entre el corazón y los pulmones es uno de los ritmos básicos de la vida y fluctúa con la frecuencia del latido cardiaco y de la respiración. Estas interacciones mecánicas entre el sistema respiratorio y el cardiovascular fueron descritas por primera vez por el fisiólogo inglés Stephen Hales que observó como el nivel de la columna de sangre de un tubo de cristal introducido en la arteria carótida de un caballo variaba de forma cíclica con la respiración (1).

El sistema cardiorrespiratorio suple de oxígeno a todo el organismo, por lo que desde un punto de vista funcional tanto el corazón como los pulmones trabajan como una misma unidad.

Esta interacción es posible debido a la localización de ambas estructuras dentro del espacio intratorácico. El corazón, en 
la práctica, es una cámara de presión localizada dentro de otra cámara de presión, el tórax. Esta es la razón por la que las variaciones de la presión intratorácica (PIT) y los cambios de volumen durante el ciclo respiratorio afectan a la función cardiaca. Además de los efectos mecánicos directos, la interacción pulmonar y cardíaca también se produce por determinados fenómenos mediados neural $\mathrm{y}$ humoralmente.

En el pulmón existe una densa red de fibras nerviosas somáticas $\mathrm{y}$ autonómicas, responsables de numerosos reflejos cardiovasculares como la arritmia sinusal respiratoria donde la insuflación de volúmenes corrientes menores de $10 \mathrm{ml} / \mathrm{kg}$ inducen un incremento de la frecuencia cardiaca debido a una disminución del tono vagal. En cambio, la ventilación con volúmenes corrientes mayores de 15 $\mathrm{ml} / \mathrm{kg}$ disminuiría la frecuencia cardiaca.

Por otra parte, la ventilación con presión positiva con o sin PEEP se ha asociado a respuestas endocrinas como la elevación de los niveles plasmáticos de noradrenalina, renina plasmática y péptido natriurético auricular.

En conjunto, estas dinámicas mecánicas, neurales y humorales constituyen la interacción corazónpulmón. $\mathrm{Su}$ conocimiento resulta fundamental cuando el clínico se enfrenta a pacientes frágiles ventilados mecánicamente, dado que estos pacientes presentan un mayor riesgo de compromiso cardiovascular, pero también para entender la monitorización hemodinámica funcional.

Bases fisiológicas para entender la interacción corazón-pulmón.
Los efectos hemodinámicos de la ventilación se pueden clasificar clínicamente en:

1. La ventilación espontánea es un ejercicio con sus demandas de gasto cardiaco y producción de $\mathrm{CO} 2$. El aporte de oxígeno a los músculos respiratorios excede con mucho sus necesidades metabólicas. En condiciones de reposo sólo se utiliza el 5\% del oxígeno para cubrir las demandas. En cambio, en determinadas condiciones patológicas como el edema pulmonar, el consumo de oxígeno puede aumentar hasta el $30 \%$. Esto podría explicar, al menos en parte, los fracasos de desconexión de la ventilación mecánica en UCI en pacientes con una reserva cardiovascular limitada.

2. La inspiración aumenta el volumen pulmonar por encima del volumen final espiratorio en reposo. Los efectos hemodinámicos de la ventilación se deben fundamentalmente a cambios en el volumen pulmonar y a la expansión de la pared torácica.

3. La inspiración espontánea disminuye la PIT, mientras que la ventilación con presión positiva la aumenta (2).

Desde un punto de vista esquemático, el sistema circulatorio consta de dos componentes principales (Fig.1):

1. Circuito: formado por el sistema vascular sistémico; arterias y vasos venosos de capacitancia.

2. Bomba: ventrículo izquierdo (VI) y ventrículo derecho (VD) rodeados por el pericardio. Debido a su localización anatómica en la fosa yuxtacardiaca rodeados por los 
pulmones y dentro de la caja torácica, son bombas que funcionan en paralelo pero que trabajan en serie $y$ que se encuentran conectadas por la circulación pulmonar.

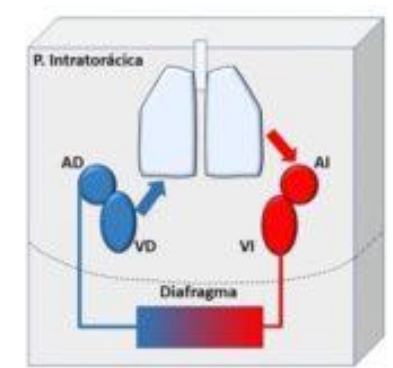

Fig. 1 Esquema del circuito cardiorrespiratorio. AD Aurícula Derecha, AI aurícula Izquierda, VD Ventrículo Derecho, VI Ventrículo Izquierdo.

Hay una serie de conceptos básicos que deben tenerse en cuenta para profundizar en la comprensión de la interacción corazón pulmón.

\section{Presión transmural}

Se entiende por presión transmural, la diferencia entre la presión dentro de una cavidad, menos la presión fuera del compartimento que se esté considerando. A modo de ejemplo, en el caso del corazón la presión transmural sería la presión que distiende las cámaras cardiacas y de algún modo la presión de trabajo contra la que tienen que contraerse (Fig. 2).

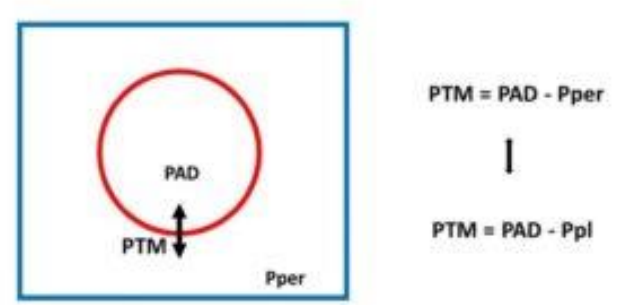

Fig. 2 Presión transmural. PAD Presión Aurícula Derecha, Pper Presión Pericárdica, Ppl Presión Pleural, PTM Presión Transmural

La presión que rodea inmediatamente al corazón es la presión pericárdica (Pper).
En ausencia de patología pericárdica, la Pper equivale a la presión pleural $(\mathrm{Ppl})$. Ésta última, varía con las diferentes fases de la respiración tanto en ventilación espontánea como en mecánica.

Las presiones medidas con los sistemas de monitorización invasiva empleados en la clínica no son absolutas sino que dependen del nivel de cero elegido; generalmente a nivel atmosférico en el 4 espacio intercostal en la línea medioaxilar (eje flebostático) (3). Esta práctica, correcta en el caso de los vasos extratorácicos, introduce un error en las mediciones de la PIT, porque la presión que rodea a las estructuras dentro del tórax no es la atmosférica sino la $\mathrm{Ppl}$ (Fig.2).

Por tanto, la presión de una cámara cardiaca sería la diferencia de presión dentro de la cavidad (relativa a la atmosférica) y la presión que la rodea o la Ppl (también relativa a la atmósfera). A modo de ejemplo la presión transmural de la AI (PAItm) sería

PAItm $=$ PAI - Ppl donde,

PAItm presión transmural de la AI

PAI presión intracavitaria de la AI

Ppl Presión pleural

Como se expuso anteriormente la Ptm es la presión de distensión real de la cámara cardiaca y es la presión de trabajo contra la cual las cámaras cardiacas deben de contraerse $\mathrm{y}$, junto con la complianza, definen el llenado y las dimensiones de cada cámara, pero también de los vasos sanguíneos.

\section{Presión media de llenado sistémico.}

Cerca del $70 \%$ del volumen sanguíneo reside en las venas. La mayor parte prácticamente no genera Ptm (volumen 
no estresado). El volumen de sangre que crea Ptm en el circuito sistémico y que por tanto colabora con la presión media de llenado sistémico (Pmsf) se denomina volumen estresado. $\mathrm{La}$ relación entre la Pmsf y el retorno venoso (RV) se puede comprender analizando la fórmula:

$\mathrm{RV}=(\mathrm{Pmsf}-\mathrm{PAD}) / \mathrm{RVR}$ donde,

\section{$\mathrm{RV}$ retorno venoso}

Pmsf presión media de llenado sistémico

PAD presión de aurícula derecha

RVR resistencia al retorno venoso

Como se deduce fácilmente, la diferencia entre el Pmsf y la PAD crea el gradiente de presión para vencer la resistencia al retorno venoso. Por tanto, cuanto mayor sea la PAD menor será el gradiente de presión, menor el RV y por ende menor el gasto cardiaco (Fig. 3).

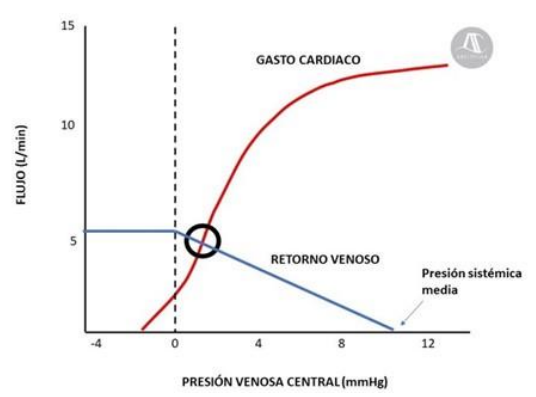

Fig. 3 Retorno venoso y precarga ventricular derecha

Pared torácica, pulmones y presiones intratorácicas.

Como anteriormente se expuso se ha asumido que la $\mathrm{Ppl}$ (medida en la práctica como presión esofágica) equivale a la Pper y ambas a la PIT. Sin embargo, realmente la PIT no se transmite de forma homogénea a través del tórax (4), especialmente ante determinadas condiciones patológicas del parénquima pulmonar (ej. derrame pleural), es más, de hecho tampoco lo hace en condiciones normales. Durante la inspiración con presión positiva los incrementos de la Ppl inducidos son menores en la zona diafragmática, debido a la distensibilidad diafragmática, y mayores en la región yuxtacardiaca.

La ventilación mecánica produce variaciones del volumen pulmonar aplicando presión por encima de la presión de retroceso elástica. Durante la fase inspiratoria de la ventilación mecánica el respirador administra un volumen corriente a los pulmones produciendo un aumento tanto de la presión alveolar (Palv) como de Ppl. La presión transpulmonar $(\mathrm{Ptp}=\mathrm{Palv}-$ Ppl) define el volumen pulmonar dependiendo de la complianza del pulmón dentro del tórax. Recordemos que la complianza pulmonar $(\mathrm{Cp})$ y de la caja torácica (Cct) definen la complianza total del sistema respiratorio (Csr) a través de la siguiente fórmula:

$1 / \mathrm{Csr}=1 / \mathrm{Cp}+1 / \mathrm{Cct}$ donde

Csr complianza del sistema respiratorio

Cp complianza pulmonar

Cct complianza de la caja torácica

Los cambios en la Palv y la Ppl por un volumen corriente dado dependerán de la Csr de tal manera que un pulmón normal transmitirá las variaciones de presión alveolar a la pleural y a las cavidades cardiacas, mientras que uno patológico amortiguará los efectos de la presión positiva de la ventilación mecánica sobre las cámaras cardiacas.

Estos cambios de volumen producen aumentos más o menos paralelos de la PIT que se transmitirán a la $\mathrm{AD}$ originando cambios dinámicos de volumen sanguíneo intratorácico durante el ciclo ventilatorio. 


\section{Interdependencia ventricular}

Los ventrículos derecho e izquierdo difieren en cuanto a su estructura anatómica y su forma de trabajar, pero presentan una interdependencia funcional derivada del hecho de trabajar en serie y estar conectados por la vascularización pulmonar, compartir septo interventricular, localizarse dentro del espacio pericárdico y a la orientación de algunas miofibrillas, de tal forma que el aumento de las presiones de llenado de un ventrículo disminuye la distensibilidad del otro (Fig. 4).

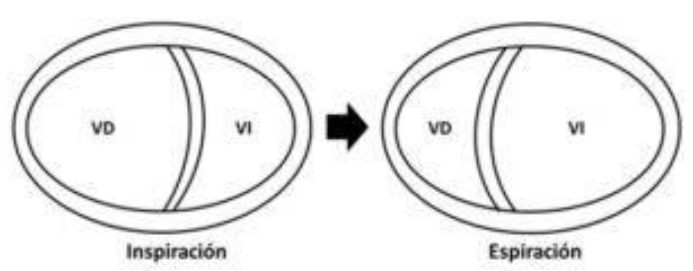

Fig.4 Interdependencia ventricular durante la ventilación espontánea. VD ventrículo derecho, VI ventrículo izquierdo.

Prueba de esta compleja interdependencia ventricular es que VD contribuye al $4-10 \%$ de la contracción izquierda, y que el VI contribuye al 20$40 \%$ de la presión sistólica del VD.

En circunstancias normales el volumen al final de la diástole y los cambios de presión al final de la diástole se modifican en direcciones opuestas en el VI $y$ en el VD durante el ciclo respiratorio. De esta forma, latido a latido, el VS que sale de los ventrículos va variando a lo largo del ciclo respiratorio, aunque en el transcurso de varios latidos la suma del VS que sale de ambos ventrículos se iguala. Este efecto fisiológico es posible debido a que tanto la vascularización pulmonar como los vasos de capacitancia son muy distensibles y permiten la acomodación del exceso de VS.

\section{Efectos de la Presión Intratorácica en la función cardiaca.}

Las relaciones establecidas entre la PIT, el retorno venoso y la función cardiaca son la base para entender las interacciones corazón-pulmón. Éstas pueden ser divididas en:

1. Efecto de la presión positiva en vía aérea en el sistema cardiovascular.

2. Efectos de los cambios cíclicos de presión en vía aérea en el sistema cardiovascular.

\section{Efecto de la presión positiva en vía aérea en el sistema cardiovascular.}

\section{Cavidades derechas.}

Como se expuso anteriormente, la presión de conducción para el retorno venoso es el gradiente entre la Pmsf y la PAD. En condiciones normales es suficiente un gradiente de presión de 4$8 \mathrm{mmHg}$ debido a que la resistencia al retorno venoso es muy baja (5). Los cambios dinámicos de la PAD durante el ciclo ventilatorio causan cambios recíprocos en el flujo venoso. Debido a que la Pmsf es prácticamente constante a lo largo de un ciclo ventilatorio, son las variaciones de la PAD las principales determinantes de la fluctuación del gradiente de presión para el retorno venoso durante la ventilación (6).

La presión transmural de la $\mathrm{AD}$, en ausencia de patología pericárdica, es la diferencia entre la PAD y la Ppl. Durante la ventilación con presión positiva, las presiones en vía aérea, $\mathrm{AD}$, pericárdicas y pleurales, se elevan al aumentar los volúmenes corrientes y la PIT de forma prácticamente lineal.

Dado que la PAD es la última presión del retorno venoso, el aumento transitorio de la PAD disminuye el 
retorno venoso del VD y disminuye el volumen sanguíneo intratorácico (Fig. $5)$.
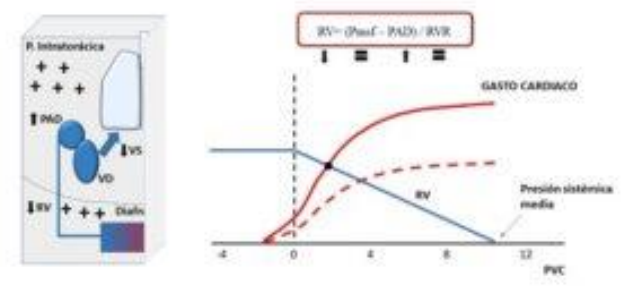

Fig 5. Efectos en el retorno venoso a cavidades derechas del aumento de la presión intratorácica durante la inspiración en ventilación mecánica. PAD presión de aurícula derecha, Pmsf presión media de llenado sistémico, PVC presión venosa central, RVR resistencias al retorno venoso, RV retorno venoso, VD ventrículo derecho, VS volumen sistólico

En pacientes con un buen estado de volemia, un efecto que mitiga, al menos en parte, la disminución de la precarga del VD es que durante la inspiración (espontánea o mecánica) se produce un descenso del diafragma que aumenta la presión intraabdominal que comprime los vasos de capacitancia abdominales (7) y se produce una traslocación de sangre desde el compartimento intratorácico hacia la circulación general.

En cambio, los pacientes deplecionados de volumen no son capaces de aumentar la Pmsf por estas vías y pueden sufrir descensos importantes del retorno venoso, el volumen sistólico y el gasto cardiaco.

Por otra parte, centrándonos en la postcarga del VD, se observa cómo el incremento de PIT tiene per se poco efecto, debido a que toda la vascularización pulmonar es intratorácica y se afecta de igual forma con los cambios de PIT. Es importante recalcar que los cambios de presión intratorácica sin cambio de volumen, no afectan al gradiente de presión entre el VD y la arteria pulmonar, ni a las resistencias vasculares pulmonares. Por tanto, ni la maniobra de Valsalva, ni los esfuerzos inspiratorios con la vía aérea obstruida (maniobra de Mueller) afectarán a la postcarga del VD. En cambio, el aumento del volumen pulmonar, mediante cambios en la distribución de zonas de West, que son los principales determinantes de la postcarga del VD, puede aumentar las resistencias vasculares pulmonares y por tanto la postcarga del VD, lo que agravaría la caída del gasto cardiaco (8).

Los vasos pulmonares tienen una complianza mucho mayor que los sistémicos, son compresibles y en la práctica se comportan como Starling resistors - un vaso que modifica su diámetro y resistencia al flujo en función de la presión que le rodea. Al aumentar la PIT, disminuye la presión transmural de la vasculatura pulmonar y aumentan las resistencias vasculares pulmonares (9).

Es conocido que la distribución del flujo en los pulmones depende de la presión arterial, venosa y alveolar. Los cambios cíclicos de presión alveolar afectan a los flujos sanguíneos regionales. En los vasos colapsables, como las venas, el flujo está estrechamente influenciado por la presión transmural. West describió 3 zonas del pulmón en el sujeto sano en bipedestación (Fig. 6).

ZONA 1 Palv $>\mathrm{Pa}>\mathrm{Pv}$ si la Palv excede tanto a la arterial como a la venosa los lechos vasculares están cerrados y no hay flujo. Habitualmente no existen zonas 1 en un pulmón sano.

ZONA 2 Pa $>$ Palv $>\mathrm{Pv}$ si la Palv se encuentra entre la $\mathrm{Pa}$ y la $\mathrm{Pv}$, existe un colapso parcial del lecho vascular y el flujo depende de la diferencia entre la $\mathrm{Pa}$ y la Pv.

ZONA 3 Pa $>$ Pv $>$ Palv si la $P v$ se encuentra entre la Pa y la Palv, el lecho vascular está completamente abierto y 
el flujo sanguíneo es continuo debido a que la presión transmural de los vasos siempre es positiva, y dependería de la diferencia entre la $\mathrm{Pa}$ y la $\mathrm{Pv}$.

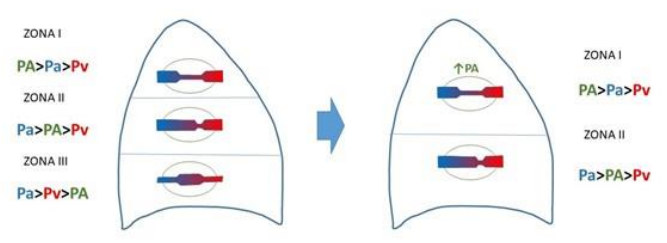

Fig. 6 Modificación de las zonas de West: izquierda en ventilación espontánea, derecha en ventilación mecánica. PA presión alveolar, $\mathrm{Pa}$ presión arterial, Pv Presión venosa.

La posición en decúbito supino y el aumento de la Palv generan el colapso de vasos alveolares (Zona 1 de West) y el incremento de la proporción de la Zona 2 en detrimento de la Zona 3. Estos cambios se traducen en un incremento de las resistencias vasculares pulmonares y del espacio muerto, que afectan a la postcarga del $\mathrm{VD}$, que es muy sensible a la insuflación cíclica con presión positiva. Esta es la razón por la cual la VM puede desencadenar un fracaso de VD en pacientes con disfunción ventricular previa o vasoconstricción hipóxica en el contexto de SDRA (10).

\section{Cavidades izquierdas}

La postcarga del VI está determinada por las resistencias vasculares periféricas (RVP) y por la presión transmural del VI (PTmvi). Como las RVP no varían significativamente a lo largo del ciclo respiratorio, son las variaciones de la PTmvi las responsables de las variaciones de la postcarga del VI.

Durante la inspiración espontánea disminuye la PIT por lo que el ventrículo tiene que contraerse con más fuerza para generar una mayor Ptm, a costa de un mayor consumo de oxígeno. Este mecanismo explicaría el estrés cardiovascular, que a menudo desencadena edema agudo de pulmón, asociado a las crisis de broncoespasmo o a los intentos de respiración contra obstrucciones de la vía aérea superior.

En cambio, durante la ventilación mecánica, la inspiración produce un aumento de la Ppl que disminuye la PTmvi y de la aorta, por lo que reduce su poscarga y facilita la eyección cardiaca (11) (Fig 7).

PTmvi = PVI-Pper donde,

PTmvi presión transmural del ventrículo izquierdo

PVI presión del ventrículo izquierdo

Pper presión pericárdica
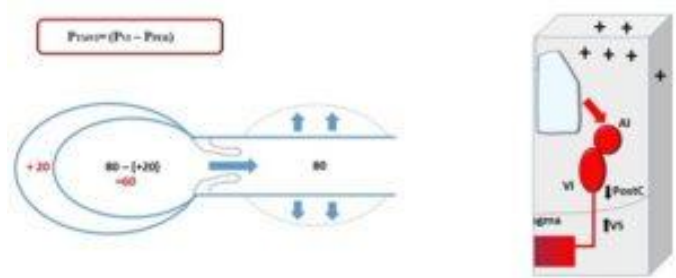

Fig. 7 Efectos del aumento de la presión intratorácica durante la ventilación mecánica en la postarga del ventrículo izquierdo. AI aurícula izquierda, PostC postcarga del ventrículo izquierdo, PTmvi presión transmural del ventrículo izquierdo, Pper presión pericárdica, PVI ventrículo izquierdo, VS volumen sistólico.

En cuanto a la precarga del VI, habitualmente la cantidad normal de sangre en la circulación pulmonar es entorno a unos $500 \mathrm{ml}: 80 \mathrm{ml}$ en las arterias pulmonares, 120 en los capilares pulmonares y 300 en las venas pulmonares.

El Aumento de PIT disminuye la Ptm de los grandes vasos intratorácicos, disminuyendo el volumen sanguíneo intratorácico que se dirige hacia el VI produciendo un aumento de su precarga.

Efectos de los cambios cíclicos de presión en vía aérea en el sistema cardiovascular. 
El efecto de la ventilación con presión positiva sobre el VS del VI tiene dos fases (Fig. 8).:

\section{1. (12).}

Durante la VM, la PIT cambia de forma cíclica y produce cambios similares a nivel de la PAD. Así, durante la inspiración aumenta la PAD con el resultado de una disminución del volumen de retorno, de la precarga y del VS del VD, y lo contrario ocurriría durante la fase espiratoria.

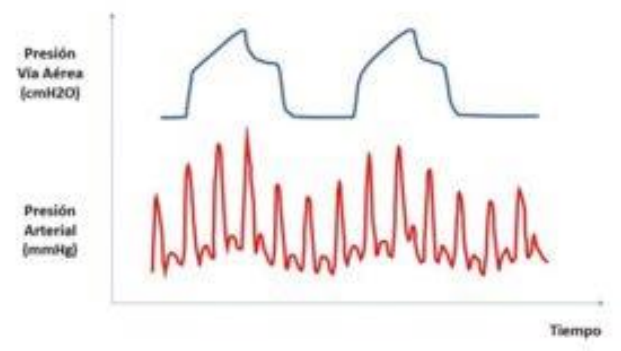

Fig. 8. Cambios cíclicos de la presión arterial invasiva durante la ventilación mecánica.

\section{Introducción a los índices dinámicos derivados de la interacción corazón pulmón}

Fueron Perel et al. y posteriormente Michard et al. los que propusieron las variaciones de amplitud de la presión arterial sistólica y de la presión de pulso respectivamente, como marcadores de hipovolemia $(13,14)$. Estos autores hipotetizaron que las variaciones inducidas por la ventilación con presión positiva están amplificadas en estados de hipovolemia debido a una reducción del volumen sanguíneo central.

La monitorización hemodinámica funcional es el empleo de determinados estresantes fisiológicos definidos para valorar la reserva fisiológica del sistema. Podría predecirse la respuesta a una carga de volumen en función de las variaciones de volumen sistólico $(\delta \mathrm{VS})$ ante determinadas variaciones de presión en vía aérea $(\delta \mathrm{P})$. De una forma excesivamente esquemática se podría afirmar que, según el estado de volemia previo, ante los mismos incrementos de PIT el paciente con adecuada volemia y por tanto "no respondedor" a carga de volumen no presentará las variaciones cíclicas de VS. En cambio, aquellos pacientes con insuficiente precarga $o$ "respondedores" tenderán a mostrar una mayor variabilidad de la PP o del VS (fig. 9).

Dicho de otro modo, en la parte ascendente de la curva de Frank Starling, las variaciones de presión intratorácica inducidas por la ventilación mecánica producirán cambios importantes del volumen sistólico. En cambio, en la parte de meseta de la curva estas variaciones serán pequeñas.

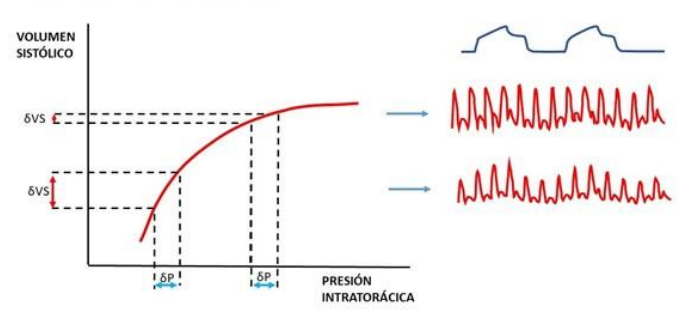

Fig. 9 Bases de la monitorización hemodinámica funcional.

Recuerde el lector que la presión de pulso, definida como la diferencia entre la PAS y la PAD es proporcional al VS e inversamente proporcional a la rigidez aórtica.

Michard demostró que la VPP con la respiración, que puede ser calculada como el porcentaje de cambio de la PP durante el ciclo respiratorio por la fórmula $\mathrm{PPV}=(\mathrm{PPmax}-\mathrm{PPmin}) /$ $[($ PPmax + PPmin $) / 2] \times 100$, podría predecir la respuesta a fluidos en pacientes en ventilación mecánica con shock séptico. Según este trabajo un valor de corte de $13 \%$ de VPP permitiría identificar aquellos pacientes precarga dependientes con un valor predictivo positivo de $94 \%$ y uno negativo de $96 \%$ (15). 
Posteriormente, el desarrollo de modelos matemáticos y algoritmos para la determinación del volumen sistólico, permitió la introducción de los monitores de análisis del contorno de la onda de pulso y se generalizó el empleo de variabilidad del volumen sistólico (VVS) en el entorno perioperatorio (16). Sin embargo, la VVS demostró ser significativamente menos precisa que la VPP (AUROC 0,84 frente a 0,94) (17), lo que probablemente sea debido a la mayor complejidad de la determinación de la VVS, que se encuentra expuesta a más errores de medición,

Durante años la aparente simplicidad en el manejo tanto de la VPP como de la VVS, despistó al clínico de una serie de importantes limitaciones. En primer lugar, hoy sabemos que el estado fisiológico normal del aparato cardiovascular es ser respondedor a cargas de volumen. Pero hay que dejar claro que esta condición no implica necesariamente la necesidad de administrar más líquidos. $\mathrm{La}$ única razón fisiológica para administrar una carga de fluido debería ser aumentar el volumen sistólico para mejorar el transporte de oxígeno.

No menos importante, la VPP y la VVS se deben a los cambios de precarga y de volumen sistólico del VD, por lo que cualquier factor que interfiera con la vascularización o función pulmonar afectará su medición. De lo que se derivan limitaciones como:

- Ventilación espontánea: no es posible establecer predicciones sobre la respuesta a volumen sobre la base de las variaciones de presión intratorácica durante la ventilación espontánea. De igual modo, también supondría una limitación el ritmo ventilatorio irregular de los pacientes ventilados en UCI con presión soporte.
- Ventilación mecánica con bajos volúmenes corrientes: Sólo un volumen tidal (VT) adecuado producirá los cambios de PIT suficientes para reducir el retorno venoso y permitir una valoración de la precarga cardiaca. De Backer en 2005 fue el primero en alertar sobre las limitaciones de los bajos VT empleados habitualmente (18). En el Síndrome de Distrés Respiratorio, aparte de por la necesidad de ventilar con bajos VT, la reducción de la complianza respiratoria disminuye la transmisión de la presión alveolar a las cavidades cardiacas lo que aumentaría los falsos negativos de estos tests. Esta limitación podría ser solventada mediante la aplicación de forma temporal de un VT de $8 \mathrm{ml} / \mathrm{kg}$ para mejorar la capacidad diagnóstica (19). Un aumento de VPP $>3,5 \%$ permitiría identificar aquellos pacientes respondedores a fluidos con una alta precisión.

- Relación frecuencia cardiacafrecuencia respiratoria $<3.6$ : Cuando la frecuencia respiratoria es alta, en torno a 16 ventilaciones por minuto, y la frecuencia cardiaca baja (unos 54 latidos por minutos), se amortiguan unos con otros los mecanismos que afectan a las variaciones cíclicas de la precarga y disminuyen las variaciones de la PP. La disminución temporal de la frecuencia ventilatoria permitiría mejorar la capacidad diagnostica de la VPP (18).

- Hipertensión intra-abdominal: Habitualmente vista en el entorno perioperatorio durante la cirugía laparoscópica o en el 
postoperatorio de cirugía abdominal mayor. El aumento de presión intraabdominal podría reducir el retorno venoso como resultado de la compresión de la vena cava y, de esta forma, podría inducir a pensar erróneamente que el paciente es respondedor a volumen. Parece que la VPP sería menos segura por la afectación de la precarga derecha $y$ de la postcarga izquierda. En cambio, algunos estudios sugieren que la VVS todavía mostraría una exactitud aceptable aunque con un punto de corte $12-14 \%(20,21)$.

- Ritmo cardiaco irregular: Como en el caso de fibrilación auricular o la extrasistolia frecuente, la VPP se debería al ritmo caótico y no tanto a las variaciones de precarga cardiaca con la ventilación mecánica. La VPP típicamente estará aumentada y no se deberá interpretar como respondedor a volumen

- Cirugía con tórax abierto: $\mathrm{Al}$ abrir el tórax desaparecería la transmisión de la presión intratorácica a la pleural por lo que característicamente estos pacientes presentarán VPP y VVS bajos a pesar de poder ser respondedores a volumen.

\section{Conclusión}

Resulta fundamental para el anestesistaintensivista, en un amplio abanico de escenarios clínicos, el conocimiento profundo de los complejos efectos hemodinámicos asociados a la ventilación mecánica. La comprensión de la interacción corazón-pulmón ayudaría a explicar, por ejemplo, la hipotensión tras la inducción anestésica y el inicio de la ventilación mecánica, los efectos positivos de la presión positiva en el paciente con insuficiencia cardiaca izquierda o el fracaso durante los intentos de destete de la ventilación mecánica. Además, la ventilación con presión positiva induce variaciones de la PP y del VS que permitiría identificar pacientes potencialmente respondedores a cargas de volumen y optimizar la fluidoterapia disminuyendo así el riesgo de sobrecarga hídrica. Por último, el clínico debe tener siempre en mente las limitaciones de la VPP y de la VVS ya que ignorarlas puede resultar en serias malinterpretaciones de estos tests.

\section{Bibliografía}

1. Sette P, Dorizzi RM, Azzini AM. Vascular access: An historical perspective from Sir William Harvey to the 1956 Nobel prize to André F. Cournand, Werner Forssmann, and Dickinson W. Richards [Internet]. Vol. 13, Journal of Vascular Access. J Vasc Access; 2012 [cited 2020 Oct 20]. p. 137-44. Available from: https://pubmed.ncbi.nlm.nih.gov/2198382 6/

2. Pinsky MR. Cardiopulmonary interactions: Physiologic basis and clinical applications. In: Annals of the American Thoracic Society. American Thoracic Society; 2018. p. S45-8. (HTML)

3. Paz-Martín D. REV ELECT ANESTESIAR-VOL 12 (6):4 Análisis de la onda de presión arterial en Anestesiología y Cuidados Intensivos I. Rev Electrónica AnestesiaR [Internet]. 2020 Sep 10 [cited 2020 Nov 15];12(6):4-4. Available from: http://revistaanestesiar.org/index.php/rear/ article/view/858

4. Lansdorp B, Hofhuizen C, Van Lavieren M, Van Swieten H, Lemson J, Van Putten MJAM, et al. Mechanical ventilation-induced intrathoracic pressure distribution and heartlung interactions. Crit Care Med. 2014;42(9):1983-90. ( PubMed)

5. Nanas S, Magder S. Adaptations of the peripheral circulation to PEEP. Am Rev Respir Dis [Internet]. 1992 [cited 2020 Oct 20];146(3):688-93. Available from: https://pubmed.ncbi.nlm.nih.gov/1519849 
6. Pinsky MR. Instantaneous venous return curves in an intact canine preparation. J Appl Physiol Respir Environ Exerc Physiol [Internet]. 1984 [cited 2020 Oct 20];56(3):765-71. Available

from: https://pubmed.ncbi.nlm.nih.gov/6368503 l

7. Chihara E, Hashimoto S, Kinoshita T, Hirose M, Tanaka Y, Morimoto T. Elevated mean systemic filling pressure due to intermittent positive- pressure ventilation. Am J Physiol - Hear Circ Physiol [Internet]. 1992 [cited 2020 Oct 20];262(4 31-4). Available from: https://pubmed.ncbi.nlm.nih.gov/1566893 I

8. Vieillard-Baron A, Matthay M, Teboul JL, Bein T, Schultz M, Magder S, et al. Experts' opinion on management of hemodynamics in ARDS patients: focus on the effects of mechanical ventilation [Internet]. Vol. 42, Intensive Care Medicine. Springer Verlag; 2016 [cited 2020 Oct 20]. p. 739-49. Available from: https://link.springer.com/article/10.1007/s 00134-016-4326-3

9. West JB, Dollery CT, Naimark A. Ditribution of blood flow in isolated lung; Relation to vascular and alveolar pressures. J Appl Physiol. 1964;19:713-24. (ㄴML)

10. Marik PE, Cavallazzi R, Vasu T, Hirani A. Dynamic changes in arterial waveform derived variables and fluid responsiveness in mechanically ventilated patients: A systematic review of the literature [Internet]. Vol. 37, Critical Care Medicine. Lippincott Williams and Wilkins; 2009 [cited 2020 Oct 24]. p. 2642-7. Available

from: https://pubmed.ncbi.nlm.nih.gov/1960297 $\underline{2 /}$

11. Pinsky MR, Summer WR, Wise RA, Permutt S, Bromberger-Barnea B. Augmentation of cardiac function by elevation of intrathoracic pressure. J Appl Physiol Respir Environ Exerc Physiol [Internet]. 1983 [cited 2020 Oct 24];54(4):950-5. Available from: https://pubmed.ncbi.nlm.nih.gov/6853301 L

12. Jardin F, Farcot JC, Gueret P, Prost JF, Ozier Y, Bourdarias JP. Cyclic changes in arterial pulse during respiratory support. Circulation [Internet]. 1983 [cited 2020 Oct 24];68(2 I):266-74. Available from: https://pubmed.ncbi.nlm.nih.gov/6861305
13. Perel A, Pizov R, Cotev S. Systolic blood pressure variation is a sensitive indicator of hypovolemia in ventilated dogs subjected to graded hemorrhage. Anesthesiology [Internet]. 1987 Oct 1 [cited 2020 Oct 3];67(4):498-502. Available

from: http://pubs.asahq.org/anesthesiology/articl e-pdf/67/4/498/315261/0000542-19871000000009.pdf

14. Michard F, Chemla D, Richard C, Wysocki M, Pinsky MR, Lecarpentier Y, et al. Clinical use of respiratory changes in arterial pulse pressure to monitor the hemodynamic effects of PEEP. Am J Respir Crit Care Med [Internet]. 1999 Mar 14 [cited 2020 Oct 26];159(3):935-9. Available from: http://www.atsjournals.org/doi/abs/10.116 $\underline{\text { 4/ajrccm.159.3.9805077 }}$

15. Michard F, Boussat S, Chemla D, Anguel $\mathrm{N}$, Mercat A, Lecarpentier Y, et al. Relation between Respiratory Changes in Arterial Pulse Pressure and Fluid Responsiveness in Septic Patients with Acute Circulatory Failure [Internet]. Vol. 162, Am J Respir Crit Care Med. 2000 [cited 2020 Oct 7]. Available from: www.atsjournals.org (HTML)

16. Hofer CK, Cecconi M, Marx G, Rocca G della. Minimally invasive haemodynamic monitoring. Eur J Anaesthesiol [Internet]. 2009 Dec [cited 2020 Oct 26];26(12):996-1002. Available

from: http://journals.lww.com/00003643$\underline{200912000-00003}$

17. Magder S, Brent G. Heart-lung interactions and pulmonary buffering: Lessons from a computational modeling study. Respir Physiol Neurobiol. 2012;182(2-3):60-70. (HTML)

18. Teboul JL, Saugel B, Cecconi M, De Backer D, Hofer CK, Monnet X, et al. Less invasive hemodynamic monitoring in critically ill patients. Intensive Care Med. 2016;42(9). (

19. Liu Y, Lou J, Mi W, Yuan W, Fu Q, Wang $M$, et al. Pulse pressure variation shows a direct linear correlation with tidal volume in anesthetized healthy patients. 2016 [cited 2020 Oct 15]; Available from: www.clinicaltrials.gov (HTML)

20. HØISETH LØ, HOFF IE, MYRE K, LANDSVERK SA, KIRKEBØEN KA. Dynamic variables of fluid responsiveness during pneumoperitoneum and laparoscopic 
surgery. Acta Anaesthesiol Scand [Internet]. 2012 Jul 1 [cited 2020 Dec 4];56(6):777-86. Available

from: http://doi.wiley.com/10.1111/j.1399_ 6576.2011.02641.x

21. Zlicar M, Novak-Jankovic V, Blagus R, Cecconi M. Predictive values of pulse pressure variation and stroke volume variation for fluid responsiveness in patients with pneumoperitoneum. J Clin Monit Comput [Internet]. 2018 Oct 1 [cited 2020 Dec 4];32(5):825-32. from: https://pubmed.ncbi.nlm.nih.gov/2914943 $\underline{3 /}$

Correspondencia al autor

Daniel Paz Martín

dpaz@anestesiar.org

Jefe de Sección Anestesiología y Reanimación. Complejo Hospitalario de Toledo.

Aceptado para el blog en febrero de 2021 